\title{
Optical properties of the human round window membrane
}

\author{
Martin Höhl \\ Daphne DeTemple \\ Stefan Lyutenski \\ Georg Leuteritz \\ Arthur Varkentin \\ Heike Andrea Schmitt \\ Thomas Lenarz \\ Bernhard Roth \\ Merve Meinhardt-Wollweber \\ Uwe Morgner
}




\title{
Optical properties of the human round window membrane
}

\author{
Martin Höhl,a,b,c,* Daphne DeTemple, ${ }^{a, b, c}$ Stefan Lyutenski, ${ }^{\mathrm{d}}$ Georg Leuteritz, ${ }^{\mathrm{b}}$ Arthur Varkentin,, \\ Heike Andrea Schmitt, ${ }^{c, d}$ Thomas Lenarz, ${ }^{c, d}$ Bernhard Roth, ${ }^{b}$ Merve Meinhardt-Wollweber, ${ }^{a, b, c}$ and \\ Uwe Morgner ${ }^{\mathrm{a}, \mathrm{b}, \mathrm{c}}$ \\ aLeibniz Universität Hannover, Institut für Quantenoptik, Hannover, Germany \\ 'Leibniz Universität Hannover, Hannoversches Zentrum für Optische Technologien, Hannover, Germany \\ "Cluster of Excellence "Hearing4all," Oldenburg, Germany \\ dMedizinische Hochschule Hannover, Experimentelle Otorhinolaryngologie, Hannover, Germany
}

\begin{abstract}
Optical techniques are effective tools for diagnostic applications in medicine and are particularly attractive for the noninvasive analysis of biological tissues and fluids in vivo. Noninvasive examinations of substances via a fiber optic probe need to consider the optical properties of biological tissues obstructing the optical path. This applies to the analysis of the human perilymph, which is located behind the round window membrane. The composition of this inner ear liquid is directly correlated to inner ear hearing loss. In this work, experimental methods for studying the optical properties of the human round window membrane ex vivo are presented. For the first time, a comprehensive investigation of this tissue is performed, including optical transmission, forward scattering, and Raman scattering. The results obtained suggest the application of visible wavelengths $(>400 \mathrm{~nm})$ for investigating the perilymph behind the round window membrane in future. $\odot 2017$ Society of Photo-Optical Instrumentation Engineers (SPIE) [DOI: 10.1117/1.JBO.22.10.105007]
\end{abstract}

Keywords: round window membrane; transmission; scattering; fiber optic probe; perilymph.

Paper 170294RR received May 5, 2017; accepted for publication Sep. 26, 2017; published online Oct. 27, 2017.

\section{Introduction}

The utilization of optical technologies in medical applications is a fast developing field of research. ${ }^{1-3}$ Noninvasive techniques for diagnostic purposes have gained much attention in the last few years. Methods such as optical coherence tomography $(\mathrm{OCT})^{4,5}$ and photoacoustic tomography ${ }^{6,7}$ are common ways to investigate biological tissues without excision or modification of properties.

However, the interaction of light with biological tissue is highly complex. ${ }^{8-10}$ Its composition and inhomogeneities lead to a strong impact (e.g., via scattering) on light propagation inside the tissue.

Another challenge is the investigation of tissues or liquids located behind a specific section of the body. The human perilymph, for example, is an inner ear liquid that is essential for hearing as it guides the mechanical waves through the cochlea to the hair cells that detect the sound waves and induce the neurological hearing sensation. It is located in the cochlea duct terminated by two membranes. On one side, there is the oval window where the auditory ossicles are connected to the cochlea. At the other side, the round window membrane (RWM) closes the cochlea duct. First insights into the protein composition of the perilymph and correlations to pathophysiologically relevant parameters pointing at diseases leading to hearing loss were reported recently. ${ }^{11,12}$ Investigations include identification of constituents in the perilymph and their concentrations as well as function. Published data are based on ex vivo measurements and limited volumes $(\sim \mu \mathrm{l})$ so far. Invasive investigations may

*Address all correspondence to: Martin Höhl, E-mail: martin.hoehl@ hot. uni-hannover.de lead to inner ear hearing loss and are only performed if a hearing impairment has already been detected. The invasive analysis of the perilymph already offered promising results concerning its diagnostic relevance. ${ }^{12}$

So far, no concept for measuring the perilymph composition noninvasively was reported. Optical methods, however, provide a large variety of noninvasive solutions for this problem. Raman spectroscopy, in particular, proved to be a valuable tool in medical diagnostics. ${ }^{13-17}$ This method was successfully utilized for analysis of the middle ear pathology. The spectral information was used to identify the calcifications prevalent in myringosclerosis. ${ }^{18}$ Furthermore, coherent anti-Stokes Raman spectroscopy microscopy was applied to display the specific molecular morphology of cholesteatoma. ${ }^{19}$ The Raman spectroscopic analysis of the perilymph requires a measurement through the RWM. Therefore, the optical properties of the RWM need to be known beforehand, which have not been measured so far either. For the efficient acquisition of Raman spectra through the RWM, the spectral transmission of the membrane needs to be determined beforehand. Furthermore, the spatial light distribution behind the membrane (forward scattering) is of major importance as it affects the excitation and collection efficiency of Raman scattered light. The Raman spectrum of the RWM itself also needs to be recorded since the excitation light transmitted across the membrane contributes to any Raman spectrum of the perilymph that is obtained and may otherwise cause artifacts.

To investigate the optical properties of human RWMs ex vivo several conditions need to be addressed. Due to the small lateral size $(\approx 1 \mathrm{~mm})$ and thickness $(70 \mu \mathrm{m})^{20}$ of human RWMs, great

1083-3668/2017/\$25.00 @ 2017 SPIE 
care must be taken in the handling of such membranes. When the transmission of small tissue samples is considered, both a tight confinement of the incident light and a corresponding collection geometry are required.

In this work, we present an experimental technique for obtaining the transmission spectra of human RWMs in the range from 250 to $1100 \mathrm{~nm}$ and discuss measured data on forward and Raman scattering. The investigations were performed ex vivo on human RWMs and pave the way for future in vivo studies.

\section{Methods and Materials}

\subsection{Experimental Setups}

\subsubsection{Transmission measurement}

The experimental setup for measuring the transmission of human RWMs is outlined in Fig. 1(a). The continuous output of a laser driven white light source (BLS, EQ-99FC-PlusUV-4-EU, Energetiq), offering a broadband spectrum (170 to $2100 \mathrm{~nm}$ ), is coupled into an optical multimode fiber (F1, UVFiber-230-2M-0022-REV4, Energetiq, core diameter of $230 \mu \mathrm{m}$ ). A reflective collimator (RC12SMA-F01, Thorlabs) directs the light further through a continuously variable neutral density filter (ND, NDC-50C-4M, Thorlabs) used for power adjustment. A second multimode fiber (F2, FG200AEA, Thorlabs, core diameter of $200 \mu \mathrm{m}$ ) collects the filtered light and guides it to the sample. The end facet is mounted on top of a third multimode fiber (F3, UM22-600, Thorlabs, core diameter of $600 \mu \mathrm{m}$ ), which is assembled inside an SMA-connector. The RWM can be placed between these opposing fibers by mounting it directly on top of the connector serving as a holder platform for the sample (S). Both fibers have a smaller core diameter (F2: $200 \mu \mathrm{m}$ and F3: $600 \mu \mathrm{m}$ ) compared to the lateral size of the RWMs measured $(\approx 1 \mathrm{~mm})$. The transmitted light is collected by the third fiber (F3) and guided to a spectrograph

(a)
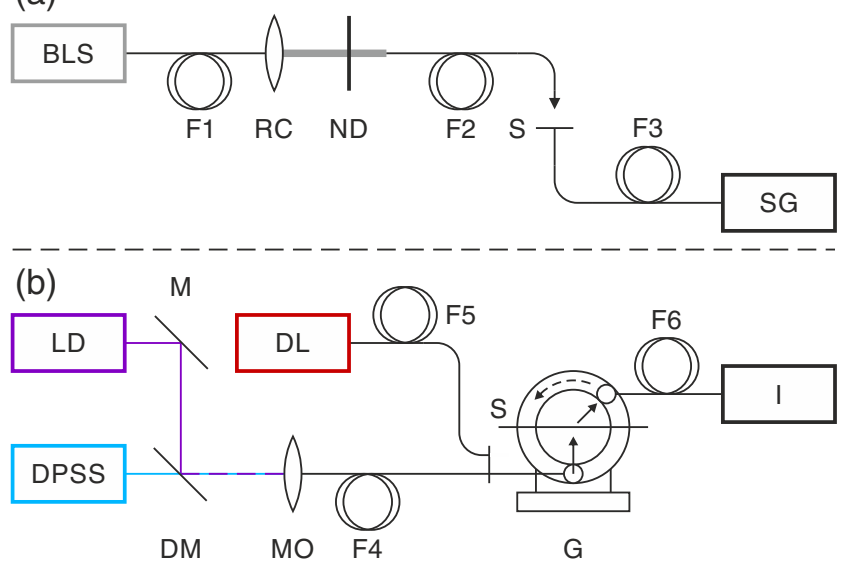

Fig. 1 The experimental setup for measuring the transmission spectra of human RWMs is shown in (a). A broadband light source (BLS, 170 to $2100 \mathrm{~nm}$ ) is applied. The sample is placed between two opposing fibers (F2 and F3). In (b), the setup for investigating the forward scattering properties of human RWMs is depicted (BLS, broadband light source; F1-6, fibers; $\mathrm{RC}$, reflective collimator; ND, neutral density filter; S, mounting platform with sample; SG, spectrograph; LD, laser diode; M, mirror; DPSS, diode-pumped solid-state laser; DM, dichroic mirror; MO, microscope objective; DL, diode laser; G, goniometer; I, integrating sphere).
(SG, Maya2000 Pro, Ocean Optics) for detection. The entrance aperture of the spectrograph has a width of $10 \mu \mathrm{m}$, and a grating with 300 lines per mm blazed for a wavelength of $300 \mathrm{~nm}$ is utilized. The spectral resolution is $0.63 \mathrm{~nm}$. Due to the low output power of the BLS and the low expected transmission of the membrane in the low wavelength region $(<400 \mathrm{~nm})$, a blaze wavelength of $300 \mathrm{~nm}$ was chosen to provide an optimal sensitivity of the spectrograph in that region.

The fiber based setup is able to obtain transmission spectra of objects that cannot be mounted in common spectrophotometers due to their size or ambient conditions (e.g., water immersion). The sample can be of arbitrary size as long as the core diameters of the excitation and detection fibers are equal to or smaller compared to the region of interest.

The transmission spectra $T(\lambda)$ were acquired corresponding to

$T(\lambda)=\frac{S(\lambda)-B(\lambda)}{R(\lambda)-B(\lambda)}$,

where $S(\lambda)$ is the spectrum of the RWM embedded in liquid phosphate buffered saline (PBS), $B(\lambda)$ the background signal when the light source is turned off, and $R(\lambda)$ the spectrum of PBS alone.

\subsubsection{Forward scattering detection}

The forward scattering properties of human RWMs were measured with the setup shown in Fig. 1(b). The scattering capability was evaluated at three different wavelengths. A laser diode (LD, CPS405, Thorlabs) operating at $404 \mathrm{~nm}$ and a diodepumped solid-state laser (DPSS, Excelsior-473-50-CDRH, Spectra-Physics) operating at $473 \mathrm{~nm}$ are combined via a dichroic mirror (DM, DMLP425R, Thorlabs). Both lasers are coupled into a fiber (F4, SM400, Thorlabs, core diameter of $2.2 \mu \mathrm{m}$ ) using a microscope objective (MO, 10X DIN SemiPlan Finite Objective, Edmund Optics) with a numerical aperture NA $=0.25$. The third wavelength $(785 \mathrm{~nm})$ is provided by a diode laser (DL, XTRA, Toptica) that is already fiber-coupled (F5, S630-HP, Thorlabs, core diameter of $3.5 \mu \mathrm{m}$ ). Both fibers (F4 and F5) provide single-mode operation for the corresponding wavelengths and guide the light to a goniometer $(\mathrm{G}, \mathrm{GON}$ 360, Instrument Systems). Depending on the wavelength, the corresponding fiber (F4 or F5) is connected to the goniometer. The light passing through the RWMs is collected by a multimode fiber (F6, OFG-312, Instrument Systems GmbH, core diameter of $600 \mu \mathrm{m})$ at different angles and guided to an integrating sphere (I, S140C, Thorlabs) for detection to determine the angle-dependent scattering function.

\subsubsection{Confocal Raman microscope}

The Raman spectra of human RWMs were measured with a confocal Raman microscope (CRM200, WITec) equipped with a standard microscope objective (LU Plan ELWD 50X, Nikon) with $N A=0.55$. For excitation, a stabilized, frequency doubled and continuous-wave Nd:YAG laser at a wavelength of $532 \mathrm{~nm}$ was used. The slit width is $50 \mu \mathrm{m}$, as determined by a multimode fiber between the Raman microscope and the spectrometer (UHTS 300, WITec). The grating used therein has 600 lines per millimeter and the spectra are detected by an emCCD camera (DU970N-BV-353, Andor). 


\subsection{Human Round Window Membranes}

\subsubsection{Availability of human samples}

The optical properties of human RWMs were determined from four samples. The number of membranes available for experiments was restricted due to several reasons. The main problem accompanying RWM isolation is the destruction of the inner ear as membrane removal opens the delicate cochlea and vestibular systems leading to loss of function.

The preferred option for determination of membrane properties would have been freshly isolated membranes of living patients. Intraoperative isolation of the RWM is performed, for example, in operations for cochlea implants. However, a resection securing a complete and especially nondamaged membrane would take a very long time exposing the patient to surplus intraoperative stress and extensive duration of narcosis with additional risks.

The other option of corpse membranes poses further difficulties. First, all corpses donated for research are routinely preserved using a mixture of formaldehyde and alcohol and set aside for several weeks before being released for experimental use. The effect of this process on biological and optical properties of the RWM, such as thickness or denaturation of proteins, varies and is impossible to be predicted in its extent. Furthermore, the number of donated corpses is usually relatively small. As isolation of the RWM destroys the inner ear, the availability is limited to any surplus specimens not used for research on inner ear diseases, which are favored due to high clinical relevance.

\subsubsection{Geometrical considerations}

The anatomy and functionality of human RWMs is described in detail elsewhere. ${ }^{20}$ The lateral size of the RWMs is $\approx 1 \mathrm{~mm}$ and was obtained from the microscopic images taken during the sample preparation process (see Fig. 2 for RWM2). For obtaining the thicknesses of the RWMs studied in this work, the samples were investigated via a spectral domain OCT system (TELESTO II, Thorlabs). The device operates at a central wavelength of $1.3 \mu \mathrm{m}$ and features a maximum imaging depth of $3.5 \mathrm{~mm}$ at an axial resolution of $4.2 \mu \mathrm{m}$ (in water). The samples were mounted on a glass slide and embedded in PBS.

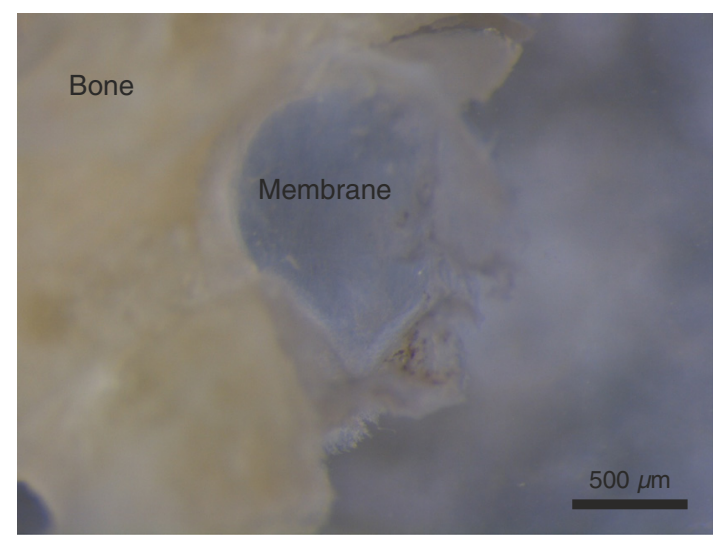

Fig. 2 Microscopic image of a human RWM, which has been extracted for measuring its optical properties ex vivo. The bony rim (that has been preserved during the preparation process) surrounds this inner ear tissue and provides membrane tension and structural stability.
A three-dimensional scan (C-scan) was obtained from each sample. Two vertical slices (B-scans) perpendicular to each other and centered in the middle of a membrane were selected for the thickness determination in the illuminated area (diameter of $200 \mu \mathrm{m}$, according to F2 for the transmission measurements). The RWM thickness was obtained at five locations (one in the middle and four at a radial distance of $100 \mu \mathrm{m}$, equally spaced). The data postprocessing and thickness determination are described in detail elsewhere. ${ }^{21}$ The results obtained for RWM1 and RWM2 are shown in Fig. 3. Optical distances (as measured by the OCT device) were transferred into geometrical distances by assuming a refractive index of 1.32 (water at $\lambda=1.3 \mu \mathrm{m}$ ). ${ }^{22}$ The calculated mean thicknesses $d$ are summarized in Table 1 . Due to the absence of the bony rims at RWM1 and RWM3, these samples lost their former tension and might be folded or swollen to a certain degree. This led to increased thicknesses measured by the OCT device, as B-scans are vertical cross sections. However, the results are in good agreement to the average RWM thickness of $70 \mu \mathrm{m} .^{20}$

\section{Results and Discussion}

\subsection{Optical Transmission of Human Round Window Membranes}

For the acquisition of transmission spectra, the extracted RWMs were aligned between the opposing fibers F2 and F3 [see Fig. 1(a)]. The spaces between sample and fibers were filled with PBS to adapt the experiment to physiological conditions. The spectral distribution of light serving as the reference $R(\lambda)$ was measured with PBS between the fibers in the wavelength range from 250 to $1100 \mathrm{~nm}$. This spectrum incorporates the spectral properties of all optical elements in the setup (response function).

The mean power applied for the subsequent measurements was $0.5 \mathrm{~mW}$ and the exposure time was set to $100 \mathrm{~ms}$. The sample spectra $S(\lambda)$, background signals $B(\lambda)$, and reference spectra $R(\lambda)$ were individually acquired three times and averaged for each sample and the spectral transmission $T(\lambda)$ was calculated according to Eq. (1). The results are shown in Fig. 4(a). The spectra were smoothed according to the spectral resolution of the spectrograph $(0.63 \mathrm{~nm})$. They show a strong extinction for wavelengths $<400 \mathrm{~nm}[T(\lambda)<16 \%]$. The absorption and scattering of UV light in biological substances lead to a low transmission in this wavelength range. ${ }^{23,24}$ Longer wavelengths are transmitted to a much higher degree $[T(\lambda>800 \mathrm{~nm})>39 \%]$. The difference among the four curves is attributed to a varying anatomy of the RWMs or different sample ages (see Table 1). The longer storage of RWM1 and the absence of the bony rim might have led to a swelling, thus, decreasing the density of molecules and increasing the transmission of this membrane. The dips in the transmission spectra of RWM3 and RWM4 are attributed to blood residues still present in the membranes as these are very recent samples. Blood vessels draw through the membrane ${ }^{20}$ and oxygenated as well as deoxygenated blood show strong absorption bands around $400 \mathrm{~nm} .{ }^{10}$

The extinction of light propagating inside a certain tissue is described by the total attenuation coefficient $\mu_{\mathrm{t}}$. The spectral dependence is characteristic for each tissue and can be used for comparing the human RWM with other tissues. It is calculated from the transmission spectrum $T(\lambda)$ and the thickness $d$ of the sample according to $\mu_{\mathrm{t}}=-(1 / d) \ln [T(\lambda)] .{ }^{25}$ From the transmission spectra shown in Fig. 4(a) and the thicknesses listed in 

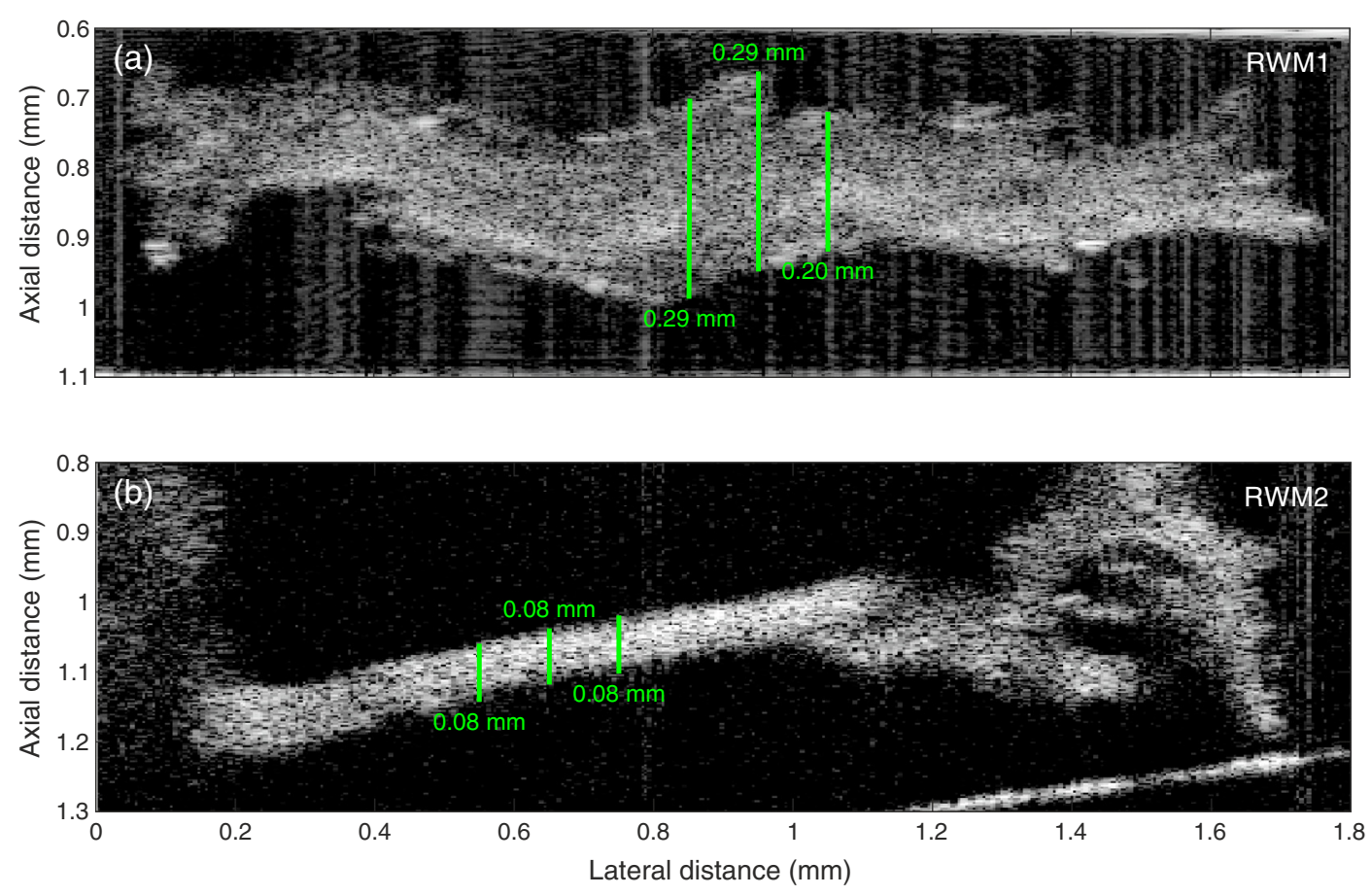

Fig. 3 OCT images (B-scans) of two different human round window membranes (RWMs). At RWM1 (a), the bony rim was removed, whereas at RWM2 (b) the rim was preserved.

Table 1 Description of the human round window membranes (RWMs) used for the subsequent optical investigations supplemented with the mean thicknesses $d$ as determined by OCT measurements (the error is given in terms of standard deviation). The measurement dates refer to the transmission spectra.

\begin{tabular}{|c|c|c|c|c|c|}
\hline Sample & Extraction date & Measurement date & Bony rim & Storage condition & $d(\mu \mathrm{m})$ \\
\hline RWM1 & January 1, 2015 & June 23, 2016 & Removed & Liquid PBS & $260 \pm 50$ \\
\hline RWM2 & April 14, 2016 & June 23, 2016 & Preserved & Frozen PBS & $76 \pm 9$ \\
\hline RWM3 & July 18,2017 & September 05, 2017 & Removed & Liquid PBS & $160 \pm 40$ \\
\hline RWM4 & July 18,2017 & September 05, 2017 & Preserved & Liquid PBS & $108 \pm 19$ \\
\hline
\end{tabular}
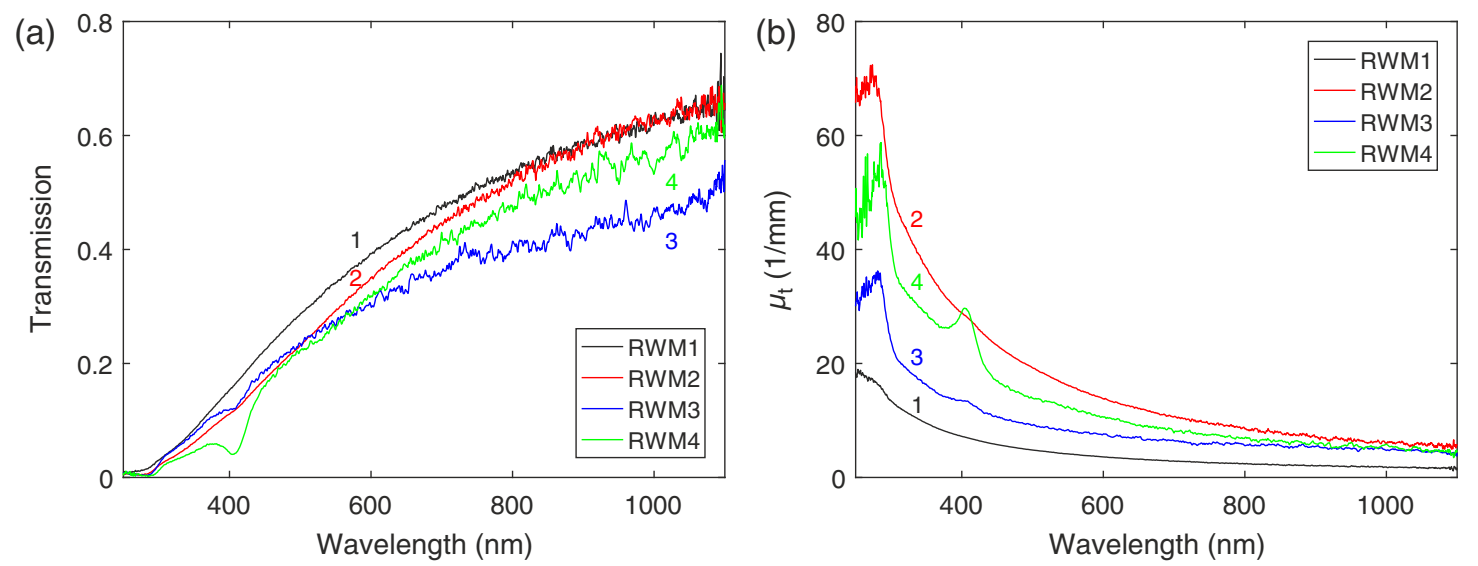

Fig. 4 The calculated transmission spectra $T(\lambda)$ of the four human round window membranes (RWMs) reveal an increasing transmission for longer wavelengths in the range from 250 to $1100 \mathrm{~nm}$ (a). There is a strong extinction of wavelengths $<400 \mathrm{~nm}$ as obvious from the total attenuation coefficient $\mu_{\mathrm{t}}$ (b). 
Table 1, the total attenuation coefficients of the four RWMs were calculated and are shown in Fig. 4(b). As expected from the transmission spectra, the attenuation decreases with increasing wavelengths. The discrepancy among the four curves is traced back to the different transmission spectra and (especially) to the varying membrane thicknesses. In the OCT measurements, larger values for the thicknesses of RWM1 and RWM3 were obtained due to the folding and swelling of these membranes. Nevertheless, the results are in good agreement with literature values obtained on other types of tissue, such as fibroglandular tissue. $^{25}$

The transmission spectra suggest the application of longer wavelengths (visible and near-infrared) for passing light through a human RWM. Especially when Raman spectra are to be recorded behind a RWM, a high transmission is essential. In this special case, the light has to cross the membrane twice (excitation light passing and Raman scattered light propagating back through the tissue).

\subsection{Forward Scattering}

The forward scattering properties of human RWMs were measured inside a goniometer [see Fig. 1(b)]. The bony rims at RWM2 and RWM4 obstructed the optical path and led to perturbing contributions in the forward scattering of these samples. In order to measure the scattering capabilities, the bony rims were removed. At RWM2, a large cut was present in the membrane afterward. Thus, it was not possible to measure the forward scattering capability of this membrane. The remaining RWMs were aligned in the focal plane of the goniometer, and the forward scattered intensity was collected in a range from $-10 \mathrm{deg}$ to $10 \mathrm{deg}$ as shown in Fig. 5(a) for RWM1. The black dots correspond to the embedding medium (PBS) and serve as the forward scattering reference. Due to its composition, the PBS shows low scattering capability. The RWMs reveal a slightly broader angular distribution (violet dots). The distributions were normalized independently from each other to restrict the comparison on the widths only. The measured intensities were fitted by a Gaussian distribution for calculating the widths $\sigma$ (indicated in terms of the FWHM). The results obtained at three different wavelengths are given in Table 2. For increasing wavelengths the angular widths decrease. The scattering cross section also decreases with increasing wavelengths leading to a narrowing of the angular distribution of scattered light. The angular width of scattered light for RWM4 at $404 \mathrm{~nm}$ is smaller compared to the width at $473 \mathrm{~nm}$. This discrepancy is traced back to the increased extinction of this membrane around $400 \mathrm{~nm}$ [see Fig. 4(b)]. The amount of forward scattered light can be described by the anisotropy factor $g$, which is calculated from the Henyey-Greenstein phase function. ${ }^{9}$ The value of $g$ ranges from -1 to 1 , where $g=1$ means that all photons are scattered in the forward direction. Due to the small thickness of human RWMs, a high value of $g$ is expected and was obtained in the measurements (see Table 2). The forward scattering properties of RWM2 are expected to be at least as beneficial as those obtained from the other RWMs, since the former is thinner and has a smoother surface due to the tension provided by the bony rim (similar to a human RWM in vivo). These results are in good agreement to the literature values for other biological tissues. ${ }^{8-10}$

Hence, a spatially confined light distribution, similar to light emanating from a fiber optic probe, can be preserved behind an RWM, which is advantageous for Raman spectroscopic measurements.

\subsection{Raman Spectroscopy}

To record the Raman spectra of the RWMs, the samples were placed in the focal plane of the microscope. Due to the small focus spot, it was possible to record Raman spectra of RWM2, in spite of the large cut. An average power of $P_{\mathrm{RWM} 1,2}=(35.60 \pm 0.18) \mathrm{mW}$ for RWM1 and RWM2, as well as $P_{\mathrm{RWM} 3,4}=(35.8 \pm 0.3) \mathrm{mW}$ for RWM3 and RWM4 was measured in front of the objective, which has a transmission of $70 \%$ at a wavelength of $\lambda=532 \mathrm{~nm}$. The exposure time was set to $t=60 \mathrm{~s}$. According to

$E=\frac{P t}{\pi r^{2}} \quad\left(r=0.61 \frac{\lambda}{\mathrm{NA}}\right)$,

the resulting energy density $E$ in the focal plane can be calculated. Here, an energy density of $E=140 \mathrm{MJ} \mathrm{cm}^{-2}$ was achieved for all measurements. The displayed Raman spectrum of each RWM was obtained by recording five spectra at different
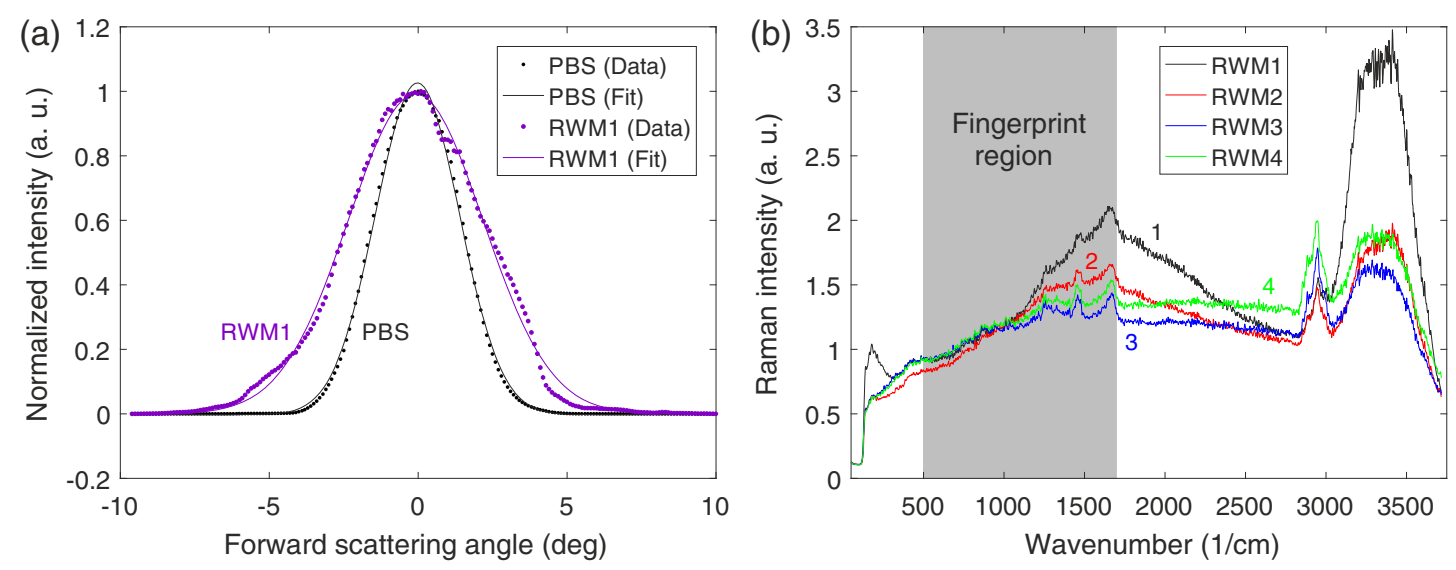

Fig. 5 The angular distribution of forward scattered light $(\lambda=404 \mathrm{~nm})$ behind a human round window membrane (RWM, violet dots) is shown in (a). The distribution is slightly broader compared to the one of PBS (black dots). The measured data were fitted by a Gaussian function (solid lines) to quantify the angular widths. (b) The mean Raman spectra of the four RWMs recorded at $532 \mathrm{~nm}$ in a confocal Raman microscope. 
Table 2 Angular widths $\sigma$ of three human RWMs and the corresponding anisotropy factors $g$ measured at different wavelengths $\lambda$. The measurements of PBS serve as the references for the RWMs. All widths are referred to the FWHM.

\begin{tabular}{lcccr}
$\lambda(\mathrm{nm})$ & $\sigma_{\mathrm{PBS}}(\mathrm{deg})$ & $\sigma_{\mathrm{RWM} 1,3,4}(\mathrm{deg})$ & $\sigma_{\mathrm{RWM} 1,3,4} / \sigma_{\mathrm{PBS}}$ & $g_{\mathrm{RWM} 1,3,4}$ \\
\hline 404 & 3.31 & $5.32,5.74,6.59$ & $1.61,1.73,1.99$ & $0.94,0.94,0.93$ \\
473 & 3.19 & $4.88,5.52,7.22$ & $1.53,1.73,2.27$ & $0.95,0.94,0.92$ \\
785 & 3.19 & $3.38,4.60,5.50$ & $1.06,1.44,1.72$ & $0.96,0.95,0.94$ \\
\hline
\end{tabular}

spatial locations inside the membrane. No data postprocessing was applied. The spectra were averaged and the resulting mean Raman spectrum for each RWM is plotted in Fig. 5(b). The characteristic Raman features of biomolecules are located in the fingerprint region ranging from 500 to $1700 \mathrm{~cm}^{-1}$. The Raman spectrum of a human RWM is expected to be similar compared to spectra obtained from collagen due to the composition of RWMs, ${ }^{20}$ which could be confirmed in our measurements. Especially the peaks in the range from 1200 to $1700 \mathrm{~cm}^{-1}$ (amide I and III bands, inside the fingerprint region) and the peak located at $2950 \mathrm{~cm}^{-1}$ (outside the fingerprint region) are in good agreement with literature values. ${ }^{26}$ The broad and intense peak signal on the right is attributed to the water content in PBS. In the case of RWM1, this peak signal is even stronger due to the swelling of the membrane and the corresponding higher water content.

Therefore, for Raman spectra measured across a human RWM, only a slight contribution of the RWM is expected in the fingerprint region (mainly amide I and III bands). In the experiment shown above, the excitation light was focused inside the RWM and the applied energy density was considerably larger compared to usual values. Many biomolecules are probed at energy densities in the range from 0.7 to $46.1 \mathrm{MJ} \mathrm{cm}^{-2}$ (at $\lambda=532 \mathrm{~nm}) .{ }^{26,27}$ Note that for the energy densities applied in our setup, we did not observe any degradation of the RWMs via the confocal Raman microscope. When Raman spectra of molecules placed behind the membrane are to be recorded, the excitation light will be focused (if applicable) across that membrane so that the influence of the membrane on the Raman spectra is expected to be negligible.

\section{Conclusion}

We presented a comprehensive study on the optical properties of human RWMs. An experimental technique on how to measure the transmission characteristics of these membranes that can be transferred to other biological tissues was presented. The results were complemented by the determination of forward and Raman scattering properties of this inner ear tissue. The use of visible and near-infrared light for optical studies through the membrane is suggested due to the high transmission and low scattering in this spectral region. Due to the weak Raman signal obtained from the RWM itself even with a high energy density applied, no perturbing contribution to any Raman spectrum obtained from the perilymph is expected. The outcome of this work suggests the usefulness of Raman spectroscopic analysis of human perilymph through the RWM using a fiber optic probe during surgery (e.g., cochlea implant).

\section{Disclosures}

Human temporal bones were acquired from autopsies of elderly subjects in line with the donation program of the institution
(Medizinische Hochschule Hannover). No conflicts of interest, financial or otherwise, are declared by the authors.

\section{Acknowledgments}

We thank Peter Erfurt and Dr. Nils Prenzler for providing additional samples. This work was supported by the DFG Cluster of Excellence EXC 1077/1 "Hearing4all."

\section{References}

1. J. C. Hebden, S. R. Arridge, and D. T. Delpy, "Optical imaging in medicine: I. Experimental techniques," Phys. Med. Biol. 42(5), 825-840 (1997).

2. C. Balas, "Review of biomedical optical imaging: a powerful, noninvasive, non-ionizing technology for improving in vivo diagnosis," Meas. Sci. Technol. 20(10), 104020 (2009).

3. M. A. Calin et al., "Optical techniques for the noninvasive diagnosis of skin cancer," J. Cancer Res. Clin. Oncol. 139(7), 1083-1104 (2013).

4. W. Drexler et al., "Optical coherence tomography today: speed, contrast, and multimodality," J. Biomed. Opt. 19(7), 071412 (2014).

5. M. A. L. M. Boone et al., "In vivo assessment of optical properties of melanocytic skin lesions and differentiation of melanoma from nonmalignant lesions by high-definition optical coherence tomography," Arch. Dermatol. Res. 308(1), 7-20 (2016).

6. C. G. A. Hoelen et al., "Three-dimensional photoacoustic imaging of blood vessels in tissue," Opt. Lett. 23(8), 648 (1998).

7. L. V. Wang and S. Hu, "Photoacoustic tomography: in vivo imaging from organelles to organs," Science 335(6075), 1458-1462 (2012).

8. A. N. Bashkatov et al., "Optical properties of human skin, subcutaneous and mucous tissues in the wavelength range from 400 to $2000 \mathrm{~nm}$," J. Phys. D: Appl. Phys. 38(15), 2543-2555 (2005).

9. T. Lister, P. A. Wright, and P. H. Chappell, "Optical properties of human skin,” J. Biomed. Opt. 17(9), 090901 (2012).

10. S. L. Jacques, "Optical properties of biological tissues: a review," Phys. Med. Biol. 58(11), R37-R61 (2013).

11. A. C. Lysaght et al., "Proteome of human perilymph," J. Proteome Res. 10(9), 3845-3851 (2011).

12. H. A. Schmitt et al., "Proteome analysis of human perilymph using an intraoperative sampling method," J. Proteome Res. 16(5), 1911-1923 (2017).

13. Q. Tu and C. Chang, "Diagnostic applications of Raman spectroscopy," Nanomed. Nanotechnol. Biol. Med. 8(5), 545-558 (2012).

14. C. Eklouh-Molinier et al., "In vivo confocal Raman microspectroscopy of the human skin: highlighting of spectral markers associated to aging via a research of correlation between Raman and biometric mechanical measurements," Anal. Bioanal. Chem. 407(27), 8363-8372 (2015).

15. F. M. Lyng et al., "Raman spectroscopy for screening and diagnosis of cervical cancer," Anal. Bioanal. Chem. 407(27), 8279-8289 (2015).

16. W. Wang et al., "Real-time in vivo cancer diagnosis using Raman spectroscopy," J. Biophotonics 8(7), 527-545 (2015).

17. H. K. Yosef et al., "In vitro prediction of the efficacy of molecularly targeted cancer therapy by Raman spectral imaging," Anal. Bioanal. Chem. 407(27), 8321-8331 (2015).

18. R. Pandey et al., "Discerning the differential molecular pathology of proliferative middle ear lesions using Raman spectroscopy," Sci. Rep. 5, 13305 (2015). 
19. J. Zou et al., "Label-free visualization of cholesteatoma in the mastoid and tympanic membrane using CARS microscopy," J. Otol. 11(3), 127133 (2016).

20. M. V. Goycoolea and L. Lundman, "Round window membrane. Structure function and permeability: a review," Microsc. Res. Tech. 36(3), 201-211 (1997).

21. A. Varkentin et al., "Comparative study of presurgical skin infiltration depth measurements of melanocytic lesions with OCT and high frequency ultrasound," J. Biophotonics 10(6-7), 854-861 (2017).

22. G. M. Hale and M. R. Querry, "Optical constants of water in the 200-nm to 200- $\mu$ m wavelength region," Appl. Opt. 12(3), 555-563 (1973).

23. A. H. Fradkin et al., "UV photodegradation of murine growth hormone: chemical analysis and immunogenicity consequences," Eur. J. Pharm. Biopharm. 87(2), 395-402 (2014).

24. A. Stylianou, D. Yova, and E. Alexandratou, "Investigation of the influence of UV irradiation on collagen thin films by AFM imaging," Mater. Sci. Eng. C 45, 455-468 (2014).

25. H. Key et al., "Optical attenuation characteristics of breast tissues at visible and near-infrared wavelengths," Phys. Med. Biol. 36(5), 579590 (1991).

26. A. Rygula et al., "Raman spectroscopy of proteins: a review," J. Raman Spectrosc. 44(8), 1061-1076 (2013).

27. A.-K. Kniggendorf et al., "Temperature-sensitive gating of hCx26: high-resolution Raman spectroscopy sheds light on conformational changes," Biomed. Opt. Express 5(7), 2054-2065 (2014).

Martin Höhl is a PhD student at the Leibniz Universität Hannover. $\mathrm{He}$ received his $\mathrm{BSc}$ and $\mathrm{MSc}$ deg in physics from Westfälische Wilhelms-Universität Münster in 2010 and 2013, respectively. His current research interests include Raman spectroscopy, optical fibers, and optical characterization

Daphne DeTemple is a MSc student at Leibniz Universität Hannover. She received her BSc degree in physics in 2016. She received her MD degree from Medizinische Hochschule Hannover (MHH) in 2016 and is currently completing her $\mathrm{PhD}$ in the Transplant Laboratory at the Department for General, Visceral and Transplant Surgery at the $\mathrm{MHH}$.

Stefan Lyutenski received his Dr. med. at the University of Leipzig. Since 2011, he has been working as ENT physician at the Medizinische Hochschule Hannover. He contributes to research projects focusing on cochlea implantation and has expert knowledge in the microdissection of the inner ear.

Georg Leuteritz received his BSc degree in physics in 2013 and his MSc degree in optical technologies in 2016. He is currently a PhD student at the Institute of Product Development at the Leibniz Universität Hannover. He investigates additive manufacturing of reflective optics for individual light distributions with regard to the optimization of printed surfaces and the implementation of multimaterial printing in optics.

Arthur Varkentin received his MSc degree in physics in 2013 from Leibniz Universität Hannover. Since 2013, he is a scientific staff member and PhD student at Hannover Centre for Optical Technologies. He is working on a project for multimodal melanoma thickness assessment focusing on optical coherence tomography.

Heike Andrea Schmitt received her Dr. rer. nat. from Leibniz Universität Hannover in 2010 after studies of food and dairy technology at the University of Applied Sciences and Arts in Hannover from 1999 until 2003. Since 2013, she has been working for the project "Improved Inner Ear Diagnostics" in the Cluster of Excellence Hearing4All with the focus of mass spectrometric analysis of perilymph.

Thomas Lenarz studied medicine and biochemistry at the universities of Tübingen, Erlangen, Heidelberg, and London from 1975 until 1981 and received his doctoral degree in medicine. He received his PhD in pharmacology of the auditory system in 1987 and worked as postdoctoral research fellow at the University of California in San Francisco in 1989. Since 1993, he has been the chairman and professor at the Department of Otolaryngology, Medizinische Hochschule Hannover.

Bernhard Roth received his $\mathrm{PhD}$ in 2001 from the University Bielefeld. From 2002 to 2007, he was the group leader at the University Düsseldorf and received his habilitation in quantum optics in 2007 . From 2007 to 2010 , he was an associate professor at the University Düsseldorf and from 2011 to 2012 managing director at the Research Centre innoFSPEC, University Potsdam, and Leibniz Institute for Astrophysics Potsdam.

Merve Meinhardt-Wollweber received her Dr. rer. nat. from Leibniz Universität Hannover in 2006. Since 2010, she leads the laser spectroscopy in life sciences team at the Hanover Centre for Optical Technologies. Her main research interests are development of spectroscopic methods for application in biomedicine and environmental analysis. The covered topics range from optoacoustics and Raman spectroscopy to illumination technology and fiber sensors.

Uwe Morgner has been a full professor with the Physics Department at Leibniz Universität Hannover in Germany since 2004. Since 2006, he has been member of the board of directors at Laser Zentrum Hannover e.V. (LZH). He has been working in the field of ultrafast lasers for more than 15 years. His major research focus is on single and few cycle pulses from lasers and parametric sources and their applications in biophotonics and fundamental science. 